\title{
ArcheoSciences
}

Revue d'archéométrie

29 | 2005

Varia

\section{Les peintures de terres cuites à Carthage. Identification des pigments de figurines et de fruits}

Tijani Karmous, Zohra Cherjf, Naceur Ayed et Claude Coupry

\section{(2) OpenEdition}

1 Journals

Édition électronique

URL : https://journals.openedition.org/archeosciences/473

DOI : 10.4000/archeosciences.473

ISBN : 978-2-7535-1594-9

ISSN : 2104-3728

Éditeur

Presses universitaires de Rennes

Édition imprimée

Date de publication : 31 décembre 2005

Pagination : $43-50$

ISSN : $1960-1360$

Référence électronique

Tijani Karmous, Zohra Cherjf, Naceur Ayed et Claude Coupry, « Les peintures de terres cuites à

Carthage. Identification des pigments de figurines et de fruits », ArcheoSciences [En ligne], 29 | 2005

mis en ligne le 31 décembre 2007, consulté le 01 février 2022. URL : http://journals.openedition.org/ archeosciences/473; DOI : https://doi.org/10.4000/archeosciences.473 


\title{
Les peintures de terres cuites à Carthage. Identification des pigments de figurines et de fruits
}

\author{
Tijani KARMOUS*, Zohra CHERIF**, Naceur AYED* et Claude COUPRY***
}

\begin{abstract}
Résumé : Le développement de l'artisanat punique à Carthage a fait l'objet de nombreuses études mais un très petit nombre concerne les matériaux utilisés dans les terres cuites et leur décor. L'article présente les analyses de pigments décorant deux types d'objets, fruits à usage funćraire et statuettes. Elles ont été réalisćes sur des micro-prélèvements, essentiellement par microspectrométrie Raman, complétée dans quelques cas par des analyses ćlémentaires. Elles montrent l'utilisation seulement de produits inorganiques. A l'époque punique, la palette de l'artiste comporte des oxydes de fer jaune et rouge, composés très largement répandus dans la nature et du bleu égypticn, comme dans l'ensemble du bassin méditerranéen. Deux pigments sont particulièrement intéressants car les matières premières ne sont pas d'origine locale : la présence de sulfure de mercure, qui pourrait provenir de la péninsule ibérique, ct le carbonate basique vert de cuivre, la malachite, qui supposc un approvisionnement en cuivrc. Les engobes blancs sont à base de kaolinite. Pour tous les engobes de fruit, un oxyde de titanc, l'anatase, a été identifié et pourrait çtre une indication d'origine géographique de la kaolinite. L'engobe d'une statuettc contient un composé phosphoré, vraisemblablement un phosphate de calcium, pour en renforcer la coulcur blanche.
\end{abstract}

\begin{abstract}
The punic craftsmanship development in Carthage was the subject of numerous studies, however, a few of them dealt with the materials used in baked ciay and its decoration. The report shows the analysis of pigments decorating two types of objects, fruits for funeral uscs and statuettes. These analysis have been achicved on microsamples, essentially by Raman micro-spectrometry and sometimes completed by ciementary ones. No organic colored product was detected. During the punic cra, the artist's palette comprises yellow and red iron oxides, components widely spread in nature, and also Egyptian blue, like the one in the Mediterranean world. Two pigments are particularly interesting because their raw materials are not from a local origin: mercury sulfide which may originate from Ibcrian peninsula and malachite which engenders the need for a supply in copper. The white coatings are kaolinit basis. For all the fruit coatings, a titanium oxide, anatasc, has been identified and might be an indication of kaolinite geographic origin. The coating of a statuette contains a phosphoruss component, which could be calcium phosphate, in order to reinforce white colour
\end{abstract}

Mots-clefs : Peinturc sur terre cuite, figurine terre cuite, colorants de Carthage, colorants puniques, analyses colorants.

Key-words: Paint on terracotta, terracotta‘s figurines, Carthage colorants, punic colorants, colorants analyses.

\section{Introduction}

Moins de deux siècles après sa fondation à la fin du IXe siècle $\mathrm{BC}$, Carthage a connu un grand développement. Une recherche récente a montré que les auteurs anciens, Hérodote, Pline... étaient unanimes pour vanter les artisans de la cité : ébénistes, bronziers, verriers, potiers (Fantar, 1999)... Dans quelques sanctuaires et des milliers de tombes puniques, on a retrouvé (Fantar, 1986) une quantité considérable de stèles votives et funéraires, de nombreux objets en céramique, des bijoux, des vases à parfums en pâte de verre irisée et même des rasoirs et des lames en bronze présentant certaines altérations occasionnées par le vieillissement.
Ce matériel archéologique permet de mesurer l'envergure prise à Carthage par divers secteurs d'artisanat (Yacoub, 1978). Des récipients destinés à des fards, des parfums et des huiles de toilette retrouvés dans les tombes suggèrent une production de parfums, de produits de toilette et permettent d'imaginer les goûts de la femme carthaginoise et l'intérêt qu'elle attachait à la mise en valeur de sa beauté (Krandel, 1978 ; Picard et Picard, 1982 ; Karmous et al., 1996a). Des fours à chaux à la périphérie des cités puniques fabriquaient le matériau destiné à différents usages dans la construction des édifices (Fantar, 1999 ; Moscati, 1976). L'activité des fours à potiers à Carthage, à Utique... est mise en évidence par les nombreux objets retrouvés dans les villes ou dans les

\footnotetext{
* Unité de Recherche 1201 de chimie industrielle, Institut National des Sciences Appliquées et de Technologie, Rue de la Terre, BP 676, TUNIS 1080 (Tunisie). Mail Ayednaceur(yahoo.fr

** Institut National du Patrimoine, 8 place du Château, I008 TUNIS, Beb Menara (Tunisie). Mail Zohracherif(@yahoo.fr

*** Laboratoire de Dynamique, Interactions et Réactivité, UMR CNRS-Université Pierre-et-Marie Curie, 2, rue Henri Dunant, 94320 THIAIS (France).

claude.coupry(a)glvt-chrs.fr
} 
tombes (Cintas, 1953 ; Moulard, 1924). A travers divers témoignages littéraires et textes épigraphiques, il est noté que Carthage possédait de véritables fabriques de tapis et de coussins brodés, de grande réputation (Gsell, 1928). Plusieurs activités étaient associées au textile, comme la teinture à la pourpre qui fut une industrie prestigieuse à Carthage (Karmous, 1996b).

Ainsi, dans Carthage l'Antique, l'artisanat pratiqué est bien développé. Cet art carthaginois a persisté en Afrique jusqu'à la fin de l'Antiquité. Les raisons de cette survie sont multiples. Tout d'abord, la lutte acharnée qui avait opposé Carthage à Rome, était essentiellement politique et l'occupation romaine n'a pas été suivie par le déracinement de la civilisation punique. La vitalité des cités puniques explique d'autre part qu'elles aient sauvegardé leurs traditions et leur art (Yacoub, 1978).

Les couleurs jouent un rôle essentiel dans l'art carthaginois. L'importance de la teinture à la pourpre en est un exemple frappant. Les pigments utilisés pour les décors ont fait l'objet de peu de travaux analytiques (Longerstay, 1988). Le but de cette étude est, au moyen de leur identification, d'étendre la connaissance du monde carthaginois.

\section{Terres cuites décorées}

Pour leur rôle prophylactique et religieux, les terres cuites font partie du mobilier funéraire dès les VIII-VIIes siècles. C'est à des époques plus tardives qu'on les découvre dans les habitations et les temples (Cherif, 1987 Cherif, 1996 ; Ferran et Pinoud, 1955). On y retrouve les mêmes sujets que dans les objets apportés aux morts. Le fournisseur de ce type de matériel est le potier du quartier. D'après les fouilles, les ateliers de potiers sont nombreux et présents dans les différents quartiers de Carthage. Ces ateliers sont organisés en secteurs dont l'un est chargé de fournir les objets culturels et les terres cuites destinées à être déposés dans les tombes auprès des morts (Picard et Picard, 1982 ; Fantar, 1993). Pratiquement toutes les villes disposaient d'ateliers de coroplathes. Kerkouane a foumi le témoignage de tels ateliers, dont l'un dépendait du grand temple (Fantar, 1986).

Les terres cuites produites par les coroplathes sont des protomées et les masques grimaçants (Picard, 1966) qui doivent effrayer celui qui ose perturber le repos des morts. Les artisans façonnent aussi des statuettes qui sont le reflet de la société dans toute sa complexité. Ils produisent dieux et déesses, prêtres et prêtresses, des femmes de la haute société comme les visages de la femme de classe moyenne dans toutes ses taches ménagères, ainsi que des scènes de la vie quotidienne. Les scènes de distraction sont également représentées, avec des acteurs, des chanteurs, des musiciens avec leurs instruments. On a aussi des fruits et légumes, des animaux sauvages et domestiques, des fruits de mer et des brûle-parfums, avec le motif particulier à Carthage de tête de femme (Chérif, 1996).

Pour animer et donner vie aux objets, le coroplathe les a rehaussés d'une polychromie. Celle-ci n'a pas toujours résisté à l'altération de plusieurs siècles de séjour dans les tombes. Certains objets ont conservé leur décor d'origine, d'autres ne présentent que des traces à peine visibles. Afin de déterminer la nature des pigments des cou- ches colorées, des microprélévements ont été effectués sur ces objets. A travers leur identification, il est possible de remonter aux matériaux, tant naturels qu'élaborés, employés pour le décor. Cette identification est importante pour les archéologues ainsi que pour les restaurateurs. En effet, la restauration de tout matériel archéologique devrait demandé une connaissance parfaite des matériaux originels.

\section{Rôle des couleurs dans les objets}

Dans l'antiquité, le rôle de la couleur est particulièrement important quand il s'agit de fabriquer en série des objets en terres cuites. L'artisan complète par le pinceau ce que le moulage n'arrive pas à exprimer. La peinture est la dernière étape comme complément indispensable pour l'achèvement de l'œuvre.

Sur les figurines des VIII-VIIes siècles BC, l'artisan pose directement les couleurs sur l'argile décolorée par une cuisson la plus complète possible. Cette technique a été maintenue à travers les siècles (Nicollo, 1984 ; Yon, 1991). On la remarque sur les terres cuites des VII-VIes siècles BC (Chérif, 1996). Parallèlement, des terres cuites de la même époque montrent l'emploi d'une autre méthode qui consiste à couvrir la surface extérieure des pièces, avant la cuisson, d'une couche épaisse d'un enduit blanc d'aspect crayeux. Il se révèle un excellent support pour l'application des couleurs.

La palette observée est très riche : on relève les couleurs rouge, rose, brun, noir, vert, jaune et blanc. La fonction de la peinture est multiple. Le coroplathe en a usé de façon variable. Il peut se contenter de souligner des éléments déjà indiqués en relief tels que les composantes d'un pectoral ou l'ornementation d'une stéphanée ou d'une pèlerine. La peinture a servi à tracer directement des contours et à représenter des détails sans qu'on les ait au préalable indiqué par un des procédés connus, relief ou incision. Le pinceau de l'artiste a, dans ce cas, un rôle créateur et contribue à élaborer la figurine en mettant en valeur l'organisation des volumes par le contraste des couleurs. C'est ainsi que s'élaborent le caractère spécifique et la valeur artistique des terres cuites de Carthage (Chérif, 1987).

Sur la face antérieure des statuettes, le peintre déploie ses talents. Il réserve le rose ou le beige pour les parties nues du corps. On trouve le noir ou le marron à l'emplacement des cheveux. Le même noir sert pour marquer les cils, les sourcils, les paupières, les prunelles et même souvent le contour des yeux. Le noir est également utilisé pour peindre le voile, pour limiter les bordures du drapé, pour souligner le contour du décor floral ou géométrique qui rehausse une tunique ou la plinthe d'un lit. Le rouge vif a servi pour marquer les lèvres ou farder les pommettes. La même teinte est observée sur la bouche entrouverte d'un acteur, la gueule d'une lionne, les chaussures, ou la branche d'un tympanon (Chérif, 1996).

Le même souci de réalisme amène l'artisan à doter les offrandes de substitution d'une couleur conforme à celle des aliments réels, qu'ils soient fruits ou légumes. Ainsi, les grenades sont-elles rouges (Chérif, 1996). Une gousse d'ail ou un oignon ne peuvent être que blancs, le citron et 
l'amande sèche décortiquée, jaunes. Signalons cependant que sur certaines terres cuites, un rayon de miel ou une grenade, seul l'enduit blanc a subsisté. Dans le cas du raisin et de la figue étudiés, à teinte actuellement bleue, on peut imaginer que cette couleur résulte d'une dégradation du (des) produit(s) utilisé(s).

\section{Matériel et méthodes}

\section{Matériel}

Le choix des objets a été guidé par leur appartenance à la période punique, datée de $814 \mathrm{BC}$ par tradition littéraire, jusqu'en $146 \mathrm{BC}$, ainsi que par leur polychromie pour couvrir au mieux les différentes teintes. A titre de comparaison, une statuette postérieure à cette période a été jointe à l'ensemble. Les tableaux 1 et 2 regroupent les objets et les localisations des prélèvements étudiés. Pour chaque objet, sont indiquées les données archéologiques : chronologie, description et provenance. Ils sont tous actuellement conservés à la réserve du Musée de Carthage.

\section{Techniques d'analyses}

Les techniques d'analyse ont été choisies à cause de la taille microscopique des échantillons. Il s'agit de la microspectrométrie Raman pour une analyse moléculaire et, pour l'analyse élémentaire, la spectrométrie à rayons $\mathrm{X}$ induits par faisceau de protons (PIXE). Par ailleurs, des images ont été obtenues par microscopie électronique à balayage couplée à la fluorescence X (MEB-DX).

\section{Conditions expérimentales}

- Spectrométrie Raman

Microspectromètre XY (Dilor)

Longueur d'onde d'excitation

$632,8 \mathrm{~nm}$ (raie rouge du laser $\mathrm{He}-\mathrm{Ne}$ )

$514,5 \mathrm{~nm}$ (raie verte du laser $\mathrm{Ar}^{+}$)

Grossissement : $x 1000$

Détecteur : CCD refroidi à l'azote liquide

Puissance mesurée au niveau de l'échantillon : $1,2 \mathrm{~mW}$ (échantillons bleus) à $0,230 \mathrm{~mW}$ (échantillons rouge vif) ; temps d'intégration : 900 secondes (engobes blancs) à 20 secondes (échantillons rouge vif).

- PIXE

Accélérateur Grand Louvre d'Analyse Elémentaire (AGLAE)

Source des particules : hydrogène

Energie d'accélérateur : $4 \mathrm{MeV}$

Détecteur : émission énergie Chart EG

\section{Résultats}

Les résultats présentés dans les tableaux 3 et 4 et relatifs à l'analyse du matériel archéologique de Carthage montrent que l'étude en spectrométrie Raman permet l'identification sans ambiguilté des pigments, à l'exception d'un seul.

Le spectre Raman (fig. 1) des prélèvements jaunes présente une bande à $388 \mathrm{~cm}^{-1}$ accompagnée de bandes de moindre intensité, en particulier à $95 \mathrm{~cm}^{-1}$. Cet ensem- ble est caractéristique de la goethite, oxyhydroxyde de fer $\alpha-\mathrm{FeO}(\mathrm{OH})$. Ce produit jaune est très répandu dans la nature et ne demande pas ou peu de préparation pour être utilisé comme pigment (Gettens et Stout, 1966). De ce fait, il est très largement utilisé à Carthage, au moins dès le VIe siècle $\mathrm{BC}$ comme sur un citron ou une amande décortiquée en terre cuite.

Il n'est pas surprenant non plus d'identifier dans les prélèvements rouges un autre dérivé du fer, l'hématite $\alpha-\mathrm{Fe}_{2} \mathrm{O}_{3}$, dont la figure 2 présente le spectre Raman. Les raisons avancées pour une large utilisation de la goethite, son abondance naturelle et l'absence de préparation, peuvent l'être également pour l'hématite. Ce pigment a été identifié sur des statuettes, datées entre le IVe et le IIle siècle (Lancel, 1982 ; Fakhfakh, 1979). La présence d'un pigment rouge à base de fer avait été mise en évidence dès 1898 par des analyses au laboratoire de la station agronomique de Tunis (Delattre, 1904). Des fragments d'hématite ont été trouvés dans des tombes à Carthage (Cintas, 1941). L'exploitation de minerais de fer à l'époque punique est attestée par la découverte d'un four de métallurgie à Byrsa (Lancel, 1982). Des mines sont connues pour la Tunisie, ainsi à Djebel Hadida dont le nom en arabe signifie «Montagne du fer » (Snoussi, 1994).

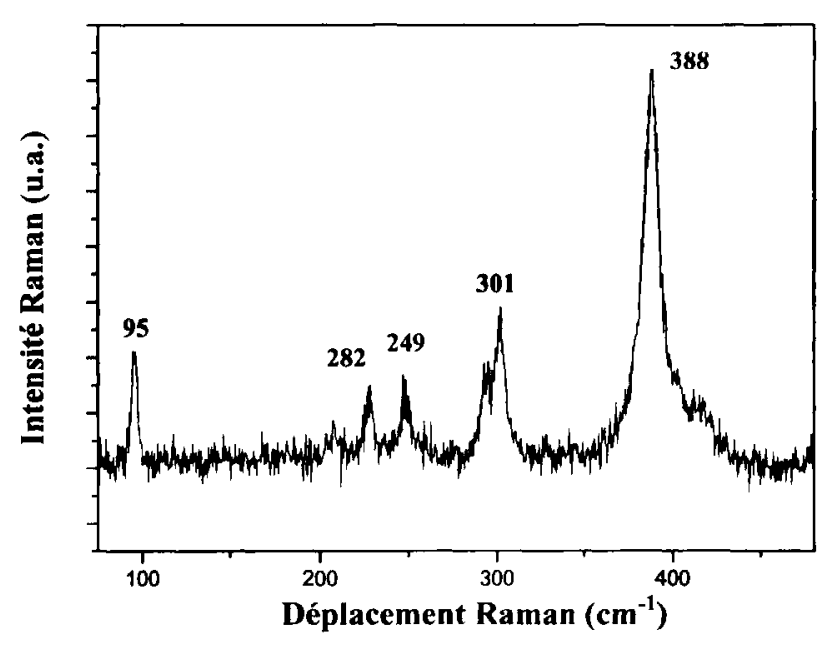

Figure 1 : Spectre Raman d'un prélèvement jaunc (amande). Figure 1: Raman Spectrum of yellow sample (Almond).

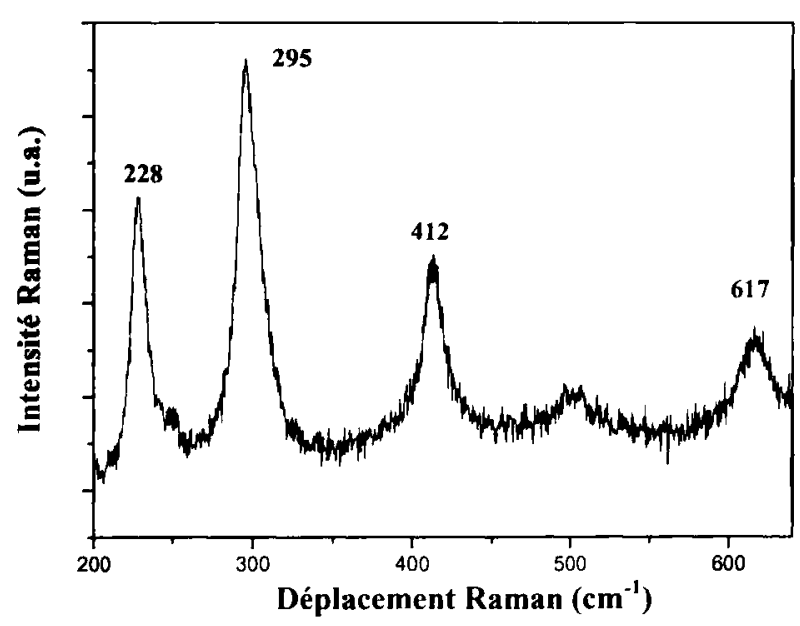

Figure 2 : Spectre Raman d'un prélèvement rouge (statuctte $n^{\circ} 2$ ), Figure 2: Raman Spectrum of red sample (Statuette $n^{\circ} 2$ ). 


\begin{tabular}{|c|c|c|c|}
\hline Statuettes & Chronologie & Bibliographie & Description * \\
\hline $\begin{array}{l}\text { Déesse joueuse de } \\
\text { tympanon }\left(n^{\circ} 1\right) \\
\text { Inventaire : CMA } 190\end{array}$ & $\begin{array}{l}\text { Fin VII }{ }^{\mathbb{e}}-\text { début } \mathrm{VI}^{\mathbf{e}} \\
\text { av. J.C. }\end{array}$ & $\begin{array}{l}\text { A Merlin B.A.C., } \\
\text { Paris }(1920) \text { pp. } 12- \\
13\end{array}$ & $\begin{array}{l}\text { Déesse au tympanon, à visage rond, sourcils marqués en noir ct la bouche en rouge } \\
\text { vif. Des disques rouges sont peints au milieu du front, sur le menton et sur chaque } \\
\text { pommette. La chevelure colorée en rouge est surmontée d'une stéphanée ornée } \\
\text { d'une rangée de rosacées bleues }\left(^{*}\right) \text { à } 8 \text { branches soulignées en noir. Le cou et le } \\
\text { haut de poitrine sont couverts d'un collier peint à } 3 \text { rangs ; le } 1^{\text {er }} \text { et le } 3^{\text {eme }} \text { sont en } \\
\text { éléments rouges, le } 2^{\text {tme }} \text { est composé de pendeloques altenativement rouges et } \\
\text { bleu-clair. Un bracelet noir à chaque poignet. Le corsage de la tunique est orné par } \\
\text { un galon, limité en noir, avec des triangles tracés en noir et peints alternativement } \\
\text { en rouge et en bleu-clair. La ceinture, à profil concave, est décorée de } \\
\text { compartiments rectangulaires accolés, et, dans chacun, des languettes verticales } \\
\text { peintes successivement en rouge et bleu-clair }\left(^{*}\right) \text {. Les franges de la ceinture sont } \\
\text { rouges limitées par une bande noire et se terminent par des effilés noirs. De la taille } \\
\text { jusqu'au bas de la tunique, tombe une bande contenant quatre rosacées bleues }\left(^{*}\right) \text { à } \\
8 \text { branches noires, rouges au coeur. } \\
\mathrm{H}: 33,3 \mathrm{~cm}\end{array}$ \\
\hline $\begin{array}{l}\text { Joueuse de double flûte } \\
\left(n^{\circ} 2\right) \\
\text { Inventaire : } 898-114\end{array}$ & $I V^{\varepsilon}-1 I^{\varepsilon}$ s. av. J.C. & $\begin{array}{l}\text { COSMOS, } 1^{\text {er }} \text { mois } \\
\text { de fouilles } \\
\text { (1898) } 816.16 \mathrm{n}^{\circ} 2\end{array}$ & $\begin{array}{l}\text { Jeune femme, debout sur une base circulaire, la tête coifféc d'une stéphanée ornéc } \\
\text { d'une branche feuillue. Elle est enveloppée dans un himation. Des deux mains, elle } \\
\text { tient une double flûte. } \\
\text { I a face antérieure est couverte d'un enduit blanc }\left(^{*}\right) \text {. Traces de rouge vif sur les } \\
\text { pieds et de bleu }\left(^{*}\right) \text { sous les bras. } \\
\text { H }: 19,3 \mathrm{~cm}\end{array}$ \\
\hline $\begin{array}{l}\text { Femme aux bras tendus } \\
\text { horizontalement }\left(\mathrm{n}^{\circ} 3\right) \\
\text { Inventaire : } 03.5\end{array}$ & $I V^{\mathrm{e}}-I I^{\mathrm{e}}$ s. av. J.C. & $\begin{array}{l}\text { Catalogue du } \\
\text { Musée Lavigerie } \\
\text { suppl. I.8.23, pl.V, } \\
3(1900)\end{array}$ & $\begin{array}{l}\text { Femme debout aux bras tendus horizontalement. La tête est enveloppée dans une } \\
\text { coiffe blcu clair, le bas du corps est enveloppé dans deux ailes croisées et serrées, } \\
\text { bleu-clair. } \\
\text { Traces d'enduit blanc, bleu-clair sur les ailes et la tête }\left(^{*}\right) \text {, rouge foncé }\left(^{*}\right) \text { sur les } \\
\text { tresses et rouge vif sur les lèvres. } \\
11: 33 \mathrm{~cm}\end{array}$ \\
\hline $\begin{array}{l}\text { Porteuse d'offrande } \\
\left(n^{\circ} 4\right) \\
\text { Inventaire } 54,1,25\end{array}$ & $I I^{\mathrm{e}}-\mathrm{I}^{\mathrm{e}}$ S. av. J.C & & $\begin{array}{l}\text { Femme debout enveloppée dans un himation qui porte des traces de couleur bleuc. } \\
\text { Traces noires sur les cheveux et les yeux,de rouge- rose }\left({ }^{*}\right) \text { sur les parties nues du } \\
\text { corps, de blanc }\left(^{*}\right) \text { sur toute la statuette. } \\
\mathrm{H}: 23,5 \mathrm{~cm}\end{array}$ \\
\hline $\begin{array}{l}\text { Tête de statuctte }\left(n^{\circ} 5\right) \\
\text { Inventaire } 16-120\end{array}$ & $I^{\varepsilon}-I I I^{\varepsilon}$ s. av. J.C. & & $\begin{array}{l}\text { Tête de jeune femme aux traits virils } \\
\text { Traces d'enduit blanc }\left({ }^{*},\right) \text { sur toute la tête, et de rouge }\left({ }^{*}\right) \text { sur le cou. } \\
\mathrm{H}: 3,8 \mathrm{~cm}\end{array}$ \\
\hline
\end{tabular}

(*) : emplacement des microprélèvements

Tableau 1 : Statuettes en terre cuite polychromées provenant du Musée de Carthage. Table 1: Polychromatic Terra cota Statuette from Carthago museum.

\begin{tabular}{|c|c|c|c|}
\hline Objets & Chronologie & Bibliographie & Description * \\
\hline Fruit : Figue 1 & IVe $-11 I^{\mathrm{E}} \mathrm{S}$. av. J.C. & $\begin{array}{l}\text { Bull. des Antiquités de France. } \\
(1904) \text { p. } 142 \text {. }\end{array}$ & $\begin{array}{l}\text { H: } 4.5 \mathrm{~cm} . \text { D: } 3.5 \mathrm{~cm} \text { Figue en terre cuite marron avec des traces d'cnduit blanc (*) } \\
\text { et de rouge-rose (*). }\end{array}$ \\
\hline Fruit: Figue 2 & {$\left[V^{5}-[]^{5} S\right.$ av. J.C. } & $\begin{array}{l}\text { Bull. des Antiquités de France. } \\
\text { (1904) p. [42.. }\end{array}$ & $\begin{array}{l}\mathrm{H}: 4.2 \mathrm{~cm} . \mathrm{D}: 3.5 \mathrm{~cm} . \\
\text { Traces d'enduit blanc }\left(^{*}\right) \text { et bleu-clair ; ainsi que des traces de rose }\left(^{*}\right) .\end{array}$ \\
\hline Fruit : Figue 3 & {$\left[V^{\varepsilon}-I I I^{s}\right.$ S. av. J.C. } & $\begin{array}{l}\text { Bull. des Antiquités de France. } \\
\text { (1904) p. 142.. }\end{array}$ & $\begin{array}{l}\mathrm{H}: 4 \mathrm{~cm} \text {. D: } 3.5 \mathrm{~cm} \text {. Cette figue présente des stries faites avant cuisson. } \\
\text { Traces d'enduit blanc (*) et de vert (*). }\end{array}$ \\
\hline Fruit: Figue 4 & $I V^{2}-I 11^{2}$ S. av. J.C. & $\begin{array}{l}\text { Bull. des Antiquités de France. } \\
\text { (1904)p. } 142 .\end{array}$ & $\begin{array}{l}\mathrm{H}: 3.5 \mathrm{~cm} . \mathrm{D}: 8 \mathrm{~cm} . \\
\text { Traces d'enduit blanc }\left(^{*}\right) \text { et de bleu. }\end{array}$ \\
\hline Fruit : Figue 5 & $I V^{\varepsilon}-I I I^{\varepsilon}$ S. av. J.C. & $\begin{array}{l}\text { Bull. des Antiquités de France. } \\
\text { (1904) p: } 142 \text {. }\end{array}$ & $\begin{array}{l}\text { H: } 5 \mathrm{~cm} . \mathrm{D}: 4.5 \mathrm{~cm} . \\
\text { Traces d'enduit blanc (*). }\end{array}$ \\
\hline Fruit : Grappe de raisin & $I V^{x}-I I I^{\subseteq} S$. av. J.C. & $\begin{array}{l}\text { Bull des Antiquités de France. } \\
\text { (1904) p. } 142 .\end{array}$ & $\begin{array}{l}\text { L: } 9.5 \mathrm{~cm} \text {. Plaque incisée } \\
\text { Traces d'enduit blanc et de bleu }\left(^{*}\right) \text {. }\end{array}$ \\
\hline Légume : Ail & $I V^{\varepsilon}-11 I^{\varepsilon}$ S. av. J.C. & $\begin{array}{l}\text { Bull. des Antiquités de France. } \\
(1904) \text { p. } 142 . .\end{array}$ & $\begin{array}{l}\mathrm{H}: 5 \mathrm{~cm} . \mathrm{D}: 3 \mathrm{~cm} . \\
\text { Enduit blanc }(*) .\end{array}$ \\
\hline Fruit : Grenade & $I^{\mathrm{e}}-\mathrm{III}^{\mathrm{e}} \mathrm{S}$. av. J.C. & $\begin{array}{l}\text { Bull. des Antiquités de France. } \\
\text { (1917)p. } 142 \text {. }\end{array}$ & $\begin{array}{l}\text { H: } 5 \mathrm{~cm} .1: 7.5 \mathrm{~cm} \\
\text { Enduit blanc }(*)\end{array}$ \\
\hline Fruit : Amande & $I^{s}=I^{s} I^{s}$ S. av. J.C. & $\begin{array}{l}\text { Bull. des Antiquités de France. } \\
\text { (1904) p. } 142 .\end{array}$ & $\begin{array}{l}\mathrm{H}: 4 \mathrm{~cm} . \\
\text { Traces de jaune }(*)\end{array}$ \\
\hline Fruit : Citron & $I V^{t}-I I I^{\mathrm{e}} \mathrm{S}$. av. J.C. & $\begin{array}{l}\text { Bull. des Antiquités de France. } \\
\text { (1904) p. } 142 \text {. }\end{array}$ & $\begin{array}{l}\text { H: } 4 \mathrm{~cm} . \text { D: } 3.5 \mathrm{~cm} \text {. } \\
\text { Traces de jaune }(*) \text {. }\end{array}$ \\
\hline $\begin{array}{l}\text { Fruit : Rayon de miel } \\
\text { (Nid d'abeille) }\end{array}$ & $I^{c}-I^{\epsilon} I^{\epsilon}$ S. av. J.C. & $\begin{array}{l}\text { Bull. des Antiquités de France. } \\
(1904) \text { p. } 142 .\end{array}$ & $\begin{array}{l}\text { H: } 7 \mathrm{~cm} \text {. L: } 4.5 \mathrm{~cm} \text {. } \\
\text { Traces d'enduit (*). }\end{array}$ \\
\hline $\begin{array}{l}\text { Boule } 1 \text { (bleu) } \\
\text { Boule } 2 \text { (bleu) } \\
\text { Boule } 3 \text { (bleu) } \\
\text { Boule } 4 \text { (bleu) } \\
\text { Boule 5 (vert) }\end{array}$ & IV $-I I^{e}$ S. av. J.C. & $\begin{array}{l}\text { Bull, des Antiquités de France. } \\
\text { (1904) p. } 142 \text {. }\end{array}$ & Boules de $1.2 \mathrm{~cm}$ de diamètre environ \\
\hline
\end{tabular}

(*) : emplacements des microprélèvements

$H$ : hauteur ; $L$ : longueur ; 1 : largeur ; D : diamètre

Tableau 2 : Objets en terre cuite polychromés provenant du Musćc de Carthage.

Table 2: Polychomatic Terra Cota Objects from Carthago museum. 


\begin{tabular}{|c|c|c|c|c|c|}
\hline \multirow{2}{*}{$\begin{array}{l}\text { I ot de } \\
\text { pigments }\end{array}$} & \multirow{2}{*}{ Support du prélèvement } & \multirow{2}{*}{$\begin{array}{c}\text { Couleur } \\
\text { du } \\
\text { pigment }\end{array}$} & \multicolumn{2}{|r|}{ Technique d'analyse } & \multirow[b]{2}{*}{ Résultats } \\
\hline & & & Technique & Données analytiques ** & \\
\hline 1 & $\begin{array}{l}\text { Ecorce d'amande décortiquée } \\
\text { Citron }\end{array}$ & Jaune & Raman & $\begin{array}{l}\text { - Raies de diffusion Raman (fig. 1): } \\
95-207-228-249-293-301-388-413 \\
\begin{array}{cccccccc}\text { M } & \text { W } & \text { M } & \text { M } & \text { W } & \text { M } & \text { S W }\end{array}\end{array}$ & La goethite \\
\hline 2 & $\begin{array}{l}\text { Statuette } 3 \text { (sur les tresses) } \\
\text { Statuette } 5 \text { (cou) }\end{array}$ & Rouge & Raman & $\begin{array}{l}\text { - Raies de diffusion Raman (fig. 2): } \\
228-248 \\
\begin{array}{llllll} & 295 & 412 & -501 & -617 \\
\text { S } & \text { W } & \text { S } & \text { M } & \text { W } & \text { M }\end{array}\end{array}$ & Hématite \\
\hline 3 & $\begin{array}{l}\text { Figue } 1 \text { (Fruit) } \\
\text { Figue } 2 \text { (Fruit) } \\
\text { Statuette } 4 \text { (Partie nue du corps) }\end{array}$ & Rose-rouge & Raman & $\begin{array}{l}\text { - Raies de diffusion Raman (fig. 3) : } \\
\begin{array}{c}44 \cdot 245 \cdots 283-344 . \\
\text { S M W W W }\end{array}\end{array}$ & Vermillon \\
\hline 4 & $\begin{array}{l}\text { Statuette I (rosacées de stéphané) } \\
\text { Statuette } 1 \text { (ceinture) } \\
\text { Statuette } 1 \text { (rosacées de la taille) } \\
\text { Statuette } 2 \text { (sous le bras) } \\
\text { Statuette } 3 \text { (tête) } \\
\text { Grappe de raisin } \\
\text { Boule 1, Boule2, Boule3,Boule4 }\end{array}$ & Bleu & Raman & 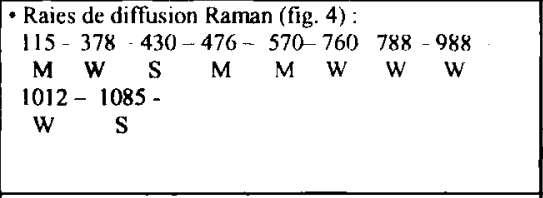 & Bleu egyptien \\
\hline 5 & Figue 3 & Verte & Raman & 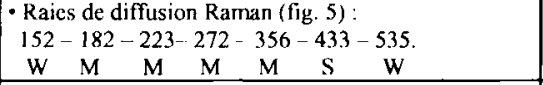 & Malachite \\
\hline 6 & Boule 5 & Verte & Raman & $\begin{array}{l}\text { - Raies de diffusion Raman (fig. 6): } \\
137-186-227-293-355-455-557-595- \\
\text { W M W M W M W W } \\
644-951.997-1084-1166 . \\
\mathrm{S} \quad \mathrm{W} \mathrm{M} \text { W M } \\
\text { Spectre MIB : Présence des éléments } \mathrm{Si}, \mathrm{AJ}, \mathrm{Ca}, \mathrm{Cu} \\
\text { accompagnés d'éléments minoritaires : } \mathrm{P}, \mathrm{K}, \mathrm{Ti} \text {. } \\
\text { Dans certaines régions, on observe une forte } \\
\text { proportion de Ti, dans d'autres du Si et Ca. }\end{array}$ & $\begin{array}{l}\text { * Les bandes Raman ainsi obtenues } \\
\text { font exclure des pigments tels que : } \\
\text { le vert de gris, le vert égyptien, la } \\
\text { malachite... } \\
\text { * La cartographie en microscopie } \\
\text { électronique montre un échantillon } \\
\text { très hétérogène dont les éléments } \\
\text { sont Si, } \mathrm{Al}, \mathrm{Ca} \text { et } \mathrm{Cu} \text {. } \\
\text { La répartition de Cu est régulière. } \\
\text { Certains grains sont riches en Ti. }\end{array}$ \\
\hline
\end{tabular}

Tableau 3 : Rćsultats des analyses des pigments de figurines ct de fruits de Carthage. Table 3: Results of analyses of pigments of terracotta figurines and fruits of Carthage.

\begin{tabular}{|c|c|c|c|c|c|}
\hline \multirow{2}{*}{$\begin{array}{l}\text { Lot de } \\
\text { pigments }\end{array}$} & \multirow{2}{*}{ Support du prélèvement } & \multirow{2}{*}{$\begin{array}{l}\text { Couleur } \\
\text { du } \\
\text { pigment }\end{array}$} & \multicolumn{2}{|r|}{ Technique d'analyse } & \multirow[b]{2}{*}{ Résultats } \\
\hline & & & $\underset{\star}{\text { Technique }}$ & Données analytiques ** & \\
\hline 7 & $\begin{array}{l}\text { Figue } 1 \\
\text { Figue } 2 \\
\text { Figue } 3 \\
\text { Figue 4 } \\
\text { Figue } 5 \\
\text { Ail } \\
\text { Grenade }\end{array}$ & $\begin{array}{l}\text { Engobe blanc } \\
\text { des fruits }\end{array}$ & $\begin{array}{l}\text { PIXE } \\
\text { MEB }\end{array}$ & 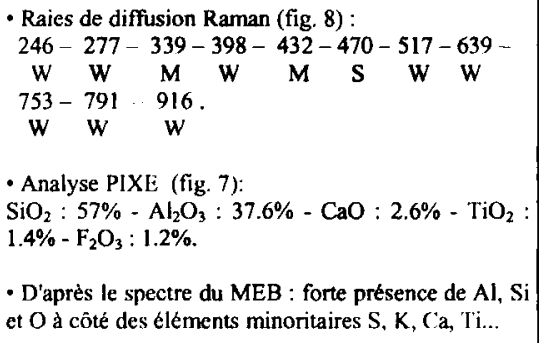 & $\begin{array}{l}\text { * Les analyses élémentaires } \\
\text { montrent la présence de } \mathrm{Si}, \mathrm{Al}, \mathrm{Ca} \text {, } \\
\text { Fe (constituants d'une argile), et Ti. } \\
\text { l.e spectre Raman présente un } \\
\text { groupc de bandes semblables à celui } \\
\text { de la kaolinite. Celle-ci contient de } \\
\text { l'oxyde de Titane (structure } \\
\text { anatase) dont les bandes de } \\
\text { diffusion caractéristiques sont } \\
\text { situées a 146,398,517 et } 639 \mathrm{~cm}^{-1} \text {. }\end{array}$ \\
\hline 8 & $\begin{array}{l}\text { Statuette I (Face) } \\
\text { Statuette } 4 \text { (sur tout le corps) } \\
\text { Statuette } 5 \text { (Tête) }\end{array}$ & $\begin{array}{l}\text { Engobe blanc } \\
\text { des } \\
\text { statuettes }\end{array}$ & Raman & $\begin{array}{l}\text { - Raies de diffusion Raman (fig. 8) : } \\
246-277-339-432 .-470-645-753-792- \\
\text { W W } \mathrm{M} \quad \mathrm{M} \quad \mathrm{S} \quad \mathrm{W} \text { W W W } \\
990 . \\
\text { S } \\
\text { - Analyse du PIXE : } \mathrm{SiO}_{2}: 37 \%-\mathrm{Al}_{2} \mathrm{O}_{3}: 31 \%- \\
\mathrm{P}_{2} \mathrm{O}_{5}: 12.9 \%-\mathrm{CaO}: 10 \%-\mathrm{Fe}_{2} \mathrm{O}_{3}: 2.2 \% \text {. }\end{array}$ & $\begin{array}{l}\text { * Les analyses élémentaires sur } \\
\text { AGLAE: montrent la présence des } \\
\text { éléments Si et Al accompagnés de } \\
\text { quantité importante de } \mathrm{P} \text { et Ca. } \\
\text { Les bandes Raman }(246,277,339, \\
\left.470,646 \text { et } 792 \mathrm{~cm}^{-1}\right) \text { sont } \\
\text { attribuables à une kaolinite } \\
\text { renfermant du phosphore caractérisé } \\
\text { par la raie intense à } 990 \mathrm{~cm}^{-1} \text {. }\end{array}$ \\
\hline
\end{tabular}

* L'attribution des raies Raman a été assurée par une base de données inteme au laboratoire du CNRS de Thiais (France)

**Intensité des raies Raman $=\mathrm{S}$ : intense, $\mathrm{M}$ : moyenne, $\mathrm{W}$; faible.

Déplacement Raman en $\mathrm{cm}^{-1}$.

Tableau 4 : Résultats des analyses des engobes de figurines et de fruits de Carthage. Table 4: Results of analyses of terracotta figurines and fruits slips of Carthage. 
Des représentations de figues des IV-IIIes siècles ont été décorées avec un pigment rouge rosâtre, sulfure de mercure $\mathrm{HgS}$, caractérisé par un spectre Raman particulièrement intense (fig. 3). Ce produit existe à l'état naturel, désigné sous le terme de cinabre. Il peut être obtenu par synthèse, appelé alors vermillon, mais ce mode de préparation semble avoir été introduit en Afrique assez tardivement. Le spectre Raman ne permet pas de distinguer l'une de l'autre origine. Ce pigment a été identifié également sur une statuette datée du début du Ile siècle BC. Des travaux antérieurs (Longerstay, 1988) avaient rapporté la présence de ce pigment sur un squelette d'une tombe carthaginoise de haute époque et concluaient, vu le manque de données sur les mines de cinabre exploitées en Afrique à l'Antiquité, à un produit importé, et provenant vraisemblablement d'Espagne.

L'identification du pigment bleu a été faite sur des prélèvements provenant de statuettes, de fruits et d'un ensemble de boules de pigment à l'état brut. Il s'agit dans tous les cas du même composé, le bleu égyptien caractérisé par le spectre Raman (fig. 4) du composant principal de sa phase cristalline, la cuprorivaite $\mathrm{CaCuSi}_{4} \mathrm{O}_{10}$. Le bleu égyptien est un pigment de synthèse élaboré dès le IIIe millénaire $\mathrm{BC}$ dans des conditions précises de tempé-

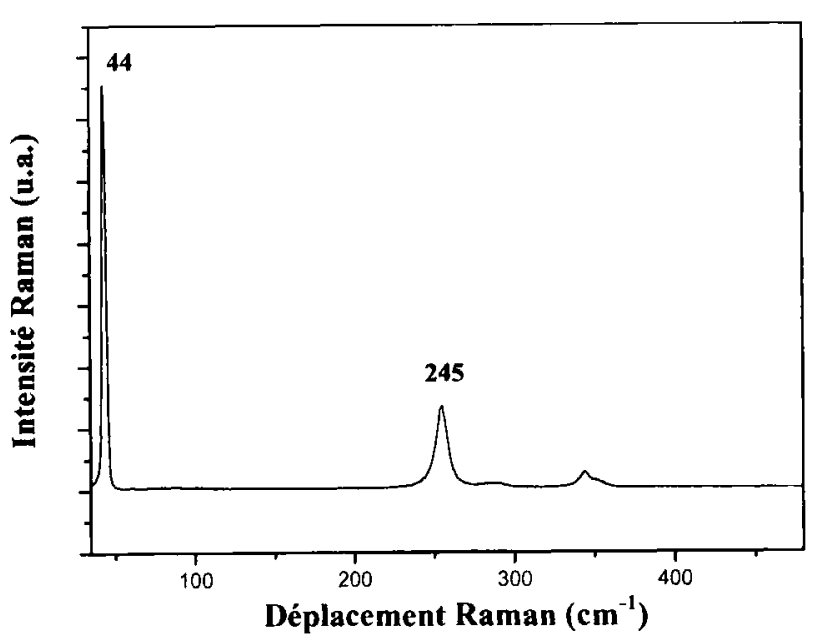

Figure 3 : Spectre Raman d'un prélèvement rouge-rose (figue 1). Figure 3: Raman Spectrum of brillant red sample (fig l).

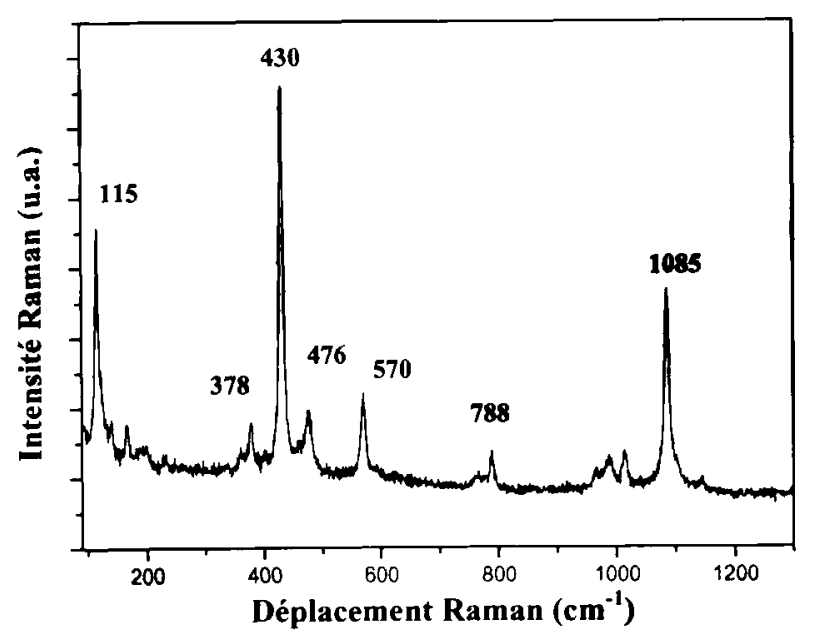

Figurc 4 : Spectre Raman d'un prélèvement bleu (raisin). Figure 4: Raman Spectrum of blue sample (grape). rature et d'atmosphère, comme l'ont montré les récentes études en archéologie expérimentale (Pagès-Camagna, 1999) dont il a fait l'objet. Il a été largement identifié dans tout le bassin méditerranéen durant toute la période antique. Nos analyses montrent que cet usage concerne également Carthage et remonte au moins au VIe siècle $\mathrm{BC}$ pour décorer le manteau d'une joueuse de tympanon. Mais s'agit-il d'un produit importé ou fabriqué localement comme le rapporte Vitruve pour Rome ? Comme la Tunisie est dépourvue de mines de cuivre, il s'agirait d'un produit importé. Cette hypothèse est confortée par les nombreuses découvertes archéologiques de boules de bleu égyptien dans des épaves en Méditerranée ainsi que par le dynamisme des commerçants carthaginois.

Un autre dérivé du cuivre, la malachite $\mathrm{CuCO}_{3} \cdot \mathrm{Cu}$ $(\mathrm{OH})_{2}$, a été identifié (fig. 5) dans une zone picturale verte sur une figue. Pour les mêmes raisons que le pigment précédent, il était vraisemblablement d'importation. Par contre, le spectre Raman (fig. 6) d'une boule de pigment vert permet d'exclure certains produits au spectre bien connu, malachite et vert-de-gris. La cartographie en microscopie électronique montre un échantillon très hétérogène dont les éléments majoritaires sont le silicium, l'aluminium, le calcium et le cuivre. La répartition du cuivre est régulière. Certains grains contiennent uniquement soit du silicium soit du calcium, d'autres de taille microscopique, 1 à $2 \mu \mathrm{m}$, sont très riches en titane. L'identification de ce pigment complexe exige l'emploi des techniques complémentaires.

Les engobes blancs sont différents et caractéristiques des deux groupes d'objets, d'une part les fruits, d'autre part, les statuettes. Les analyses élémentaires (tab. 3) montrent pour les deux groupes la présence de silicium, aluminium, calcium ainsi que d'une faible quantité de fer, accompagnés de titane, uniquement pour les fruits, et de phosphore pour une statuette. Les spectres Raman (fig. 7) correspondants présentent un groupe de bandes commun aux spectres de tous les objets, à 246, 277, 339, 432, 470,753 et $792 \mathrm{~cm}^{-1}$, semblable à celui de la kaolinite (Herman et al., 1985). De plus, les spectres de tous les fruits indiquent la présence de l'oxyde de titane, structure anatase, alors que la raie supplémentaire la plus intense

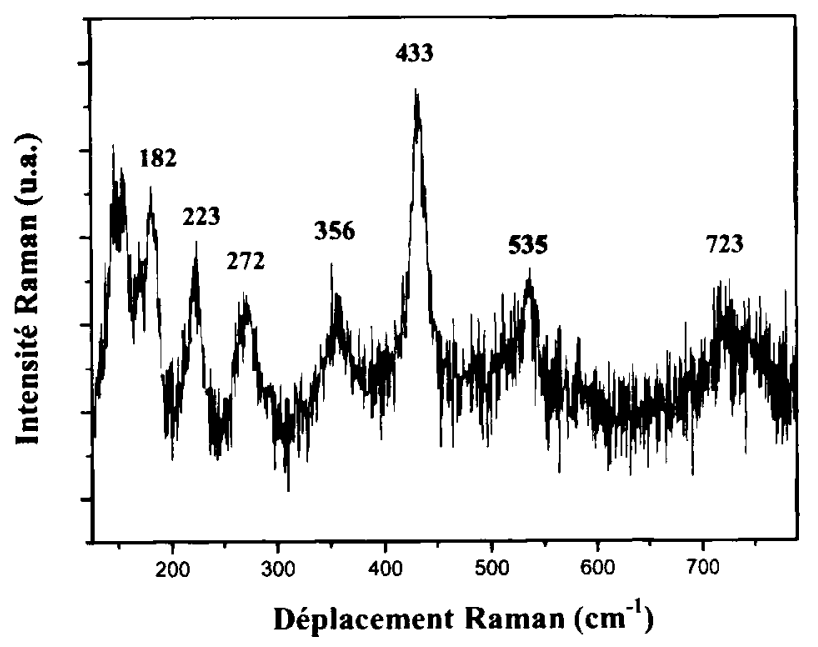

Figure 5 : Spectre Raman d'un prélèvement vert (figue 3). Figure 5: Raman Spectrum of green sample (fig 3). 


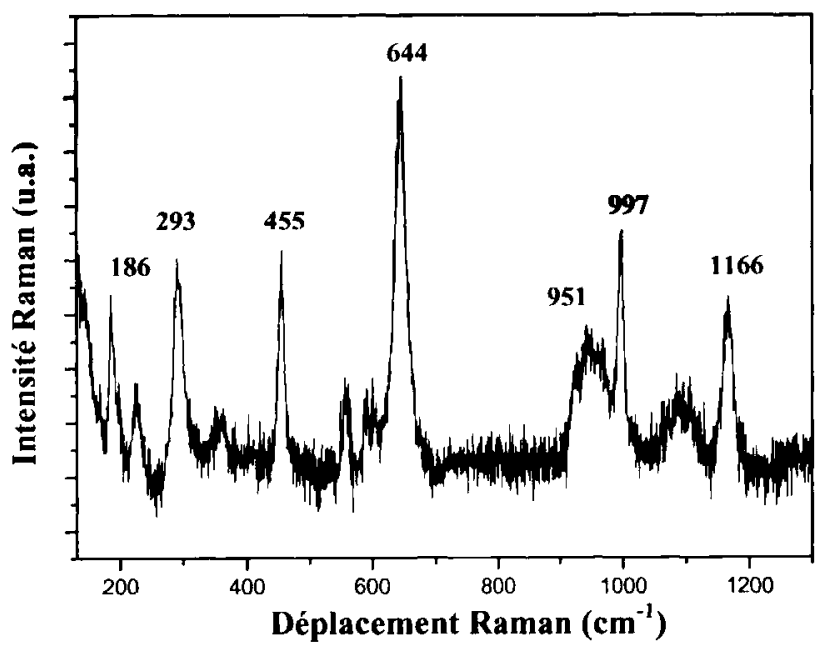

Figure 6 : Spectre Raman d'un prélèvement sur la boule verte. Figure 6: Raman Spectrum of green bawl sample.

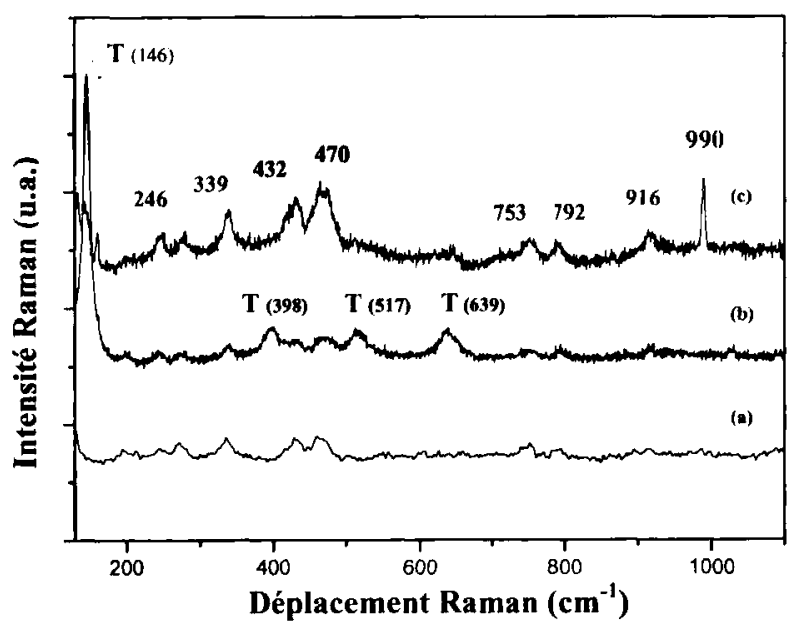

Figure 7 : Spectres Raman d'engobes blancs a) : argile kaolin (statuette $\mathrm{n}^{\circ} 2$ ) ; b) : associćc à l'anatase (ail); c) : associée à un composé phosphorć (statuctte $n^{\circ} 4$ ).

Figure 7: Raman Spectrum of white coatings samples a): Clay (Statuette $\left.n^{\circ} 2\right) ;$ b): Associated to anatase (garlic); c): associated to a phosphorus compound (Statuette $n^{\circ} 4$ ).

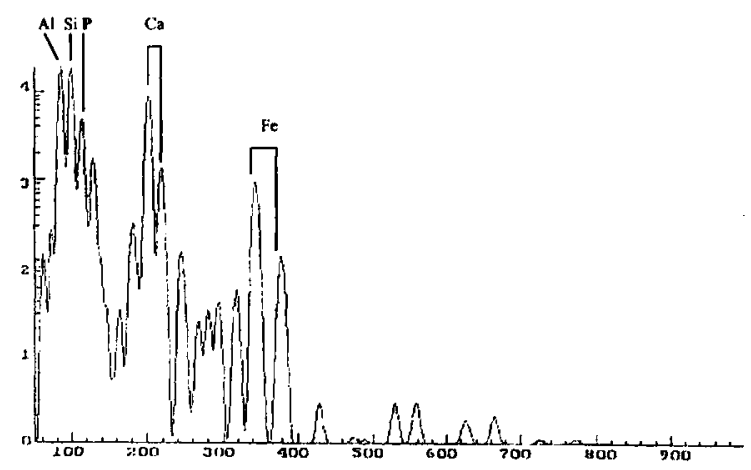

Figure 8 : Analyse élémentaire globale d'un prélèvement d'engobe blanc (statuette $\mathrm{n}^{\circ} 4$ ).

Figure 8: Global Elemenraty Analysis of white coating (Statuette $n^{\circ} 4$ ). dans le spectre de la statuette, à $990 \mathrm{~cm}^{-1}$, serait en accord avec la présence d'un phosphate de calcium d'autant que les analyses sur AGLAE montrent une quantité importante de cet élément ainsi que de calcium (fig. 8). Ce phosphate pourrait provenir d'apatite naturelle dans l'engobe, comme il pourrait correspondre à de l'os brûlé ou à une migration par décomposition d'ossements se trouvant dans la tombe. L'oxyde de titane et l'os brûlé sont des composés blancs, ajoutés peut-être pour corriger la teinte de l'argile de base, colorée par la faible quantité de fer qu'elle contient.

\section{Conclusion}

La gamme des produits identifiés sur les quelques objets étudiés ne comprend que des produits minéraux alors qu'une des techniques utilisées, la spectrométrie Raman, aurait permis d'identifier des produits organiques (Coupry et al., 1997). Par ailleurs, la pourpre (Karmous, 1996a) dont on connaît le large usage par les teinturiers à Carthage et la laque de garance sous forme de cônes de fards cosmétiques (Karmous, 1996a) ont été identifiés par HPLC. Ce dernier colorant a servi également pour décorer des objets archéologiques en terres cuites.

Le peintre fait appel à des ressources locales pour les couleurs jaunes et rouges à base de composés de fer mais n'hésite pas à recourir à des produits d'origine extérieure pour enrichir sa palette : cinabre, bleu égyptien et malachite, qui ont pu être importés sous forme de produits finis ou de minerai. L'usage du bleu égyptien se situe dans un contexte méditerranéen, celui du cinabre plutôt dans un contexte de Méditerranée occidentale, en relation avec l'expansion carthaginoise vers les nombreuses mines d'Espagne.

Tout au long de l'époque punique, les mêmes pigments ont été utilisés, quelque soit le type de support, figurines ou fruits. Par contre, la nature de l'engobe blanc, support des couches picturales, montre des variations selon les objets : kaolinite avec ou sans oxyde de titane. La présence de cet oxyde est fréquent dans les kaolinites et pourrait être un indice de provenance. L'engobe de la seule statuette II-le siècle présente une composition légèrement différente avec présence d'un composé phosphoré avec la même argile de base.

Il serait intéressant de poursuivre et d'affiner ces résultats par l'étude d'autres objets et/ou d'autres couleurs, comme la couleur noire.

\section{Remerciements}

Le conservateur du Musée de Carthage, Monsieur Abdelmajid ENNABLI, a bien voulu nous autoriser à prélever des micro-échantillons. Qu'il trouve ici l'expression de notre profonde gratitude. Les auteurs remercient très vivement Madame Sylvie Colinart (Centre de Restauration et de Recherche des Musées de France) pour les analyses qu'elle a réalisées sur AGLAE. 


\section{Bibliographie}

CHERIF, Z., 1987 - Les bijoux Carthaginois d'après les figurines en terTc cuitc. REPPAL, III 117-150.

CHERIF, Z., 1996 - Les figurines en terre cuite : Art et authenticitć. In L'Africa Romana Cartagine, Rome, 1073-1084.

CINTAS, P., 1941 - Smirat. Revue Tunisienne. 108 et 110-118.

CINTAS, P., 1953 - La ville punique au Cap-Bon en Tunisie. Comptes Renchus de l'Académie des Inscriptions des Belles Lettres, Paris, p. 258.

COUPRY, C., SAGON, G., GORGUET-BALLESTEROS, P., 1997 Raman Spectroscopic Investigation of Blue Contemporary Textiles. J. of Raman Spectrosc., 28, 85-89.

DELATTRE, A. L., 1897 - La Nécropole punique de Douimes. Bull de la société des Antiquités de France.

DELATTRE, A.L., 1904 - Les grands sarcophages anthropoiddes du Musée Lavigerie à Carthage. Cosmos. Paris.

DELATTRE, P., 1906 - Nécropole des Rab, prêtres et prêtresses de Carthage, 3ème année de fouilles. Cosmos, Paris, 16.

FAKHFAKH, M., 1979 - Les Atlas Jeune Afrique. 52.

FANTAR, MH., 1986 - Kerkouane, Citć punique du Cap Bon (Tunisic). Instirut National d'Archéologie et d'Art, Tunis, Tome III, 515-530.

FANTAR, MH., 1993 - Carthage, Approche de civilisation. Tunis, 299-302.

FANTAR, MH., 1999 - Carthage, Les Lettres et les Arts. Les éditions de la Méditerranée "Alif», Tunis, 71 et suiv.

FERRAN, J., PINOUD, M., 1955 - Fouilles de Byrsa. Cahiers de Byrsa, $\mathrm{V}$.

GAUCKLER, P., 1959 - Nécropoles Puniques. Tab. 324, p. 152 ; Tab. 476 , p. 159 ; Tab. 311, p. 134, Paris.

GETTENS, R.J., STOUT, G.L., 1966 - Painting Materials. A Short Encyclopaedia. Dover Publications, Inc. New-York.

GSELL, S., 1928 - Histoire Ancienne de l'Afrique du Nord. Hachette, Paris, Tome IV, 101-104.

HERMAN, R.G., BOGDAN, C.E. and SOMMER, A.J., 1985 - Laser Raman Microprobe Study of the Identification and Thermal Trans- formation of some Carbonate and Aluminosilicate minerals. Mater Sci. Res. 19, 113-130.

KARMOUS, T., AYED, N., FANTAR, MH., WOUTERS, J., 1996a Purple earth from Zembra and make-up from Carthage (Cartago, Tunisia). Dyes in History and Archaeology, 14, 3-8.

KARMOUS, T., AYED, N., CHEIBI, F., EL HILI, A., 1996b - La pourpre de l'ère punique : Analyse et extraction de ce colorant. Techne, 4, 57-67.

KRANDEL, A., 1978 - Documents pour l'étude de l'artisanat punique à Carthage. DEA Faculté des lettres ct Sciences Humaines, Tunis.

LANCEL, S., 1982 - Byrsa I/, Rome, 231-233.

LANCEL, S., THUILLIER, P., 1979 - Byrsa I. Romc, 256-258.

LONGERSTAY, M., 1988 - Les peintures rupestres des Haouanet de Khroumiri ct des Mogods. Actes du Xlème Séminaire de A.F.P.M.A., Rcims, 119-131.

MOSCATI, S., 1976 - I Finici e Cartagine. Societa e Costume. Torino, $490 \mathrm{p}$.

MOULARD, J., 1924 - Bull. du Comité des Travaux Historiques et Scientifiques (sec. Archéologiques). Paris, 241.

NICOLLO, R., 1984 - La fabrication des terres cuites. Dossiers de l'Archéologie, 27.

PAGĖS-CAMAGNA, S., 1999 - Propriétés physico-chimiques d'un pigment vert synthétique égyptien. Couleur, structure, recherche des techniques d'élaboration. Thèse, Université de Mamc-la-Vallćc.

PICARD, C., 1966 - Sacra Punica, les masques et les protomes. Karthago XIII, 9-3I.

PICARD, C., PICARD, G.C., 1982 - La vie quotidienne à Carthage au temps d'Hannibal. Hachettc, Paris 2ème Ed. 131-134.

SNOUSSI, S., 1994 - Ftat des Travaux réalisés par l'Office National des Mines depuis 1970 à 1992. Département de la recherche minière, Division inventaire.

YACOUB, M., 1978 - Chefs d'reuvre des musées nationaux de Tunis. Maison Tunisienne d'Edition, Tunis, 9-11.

YON, M., 1991 - Techniques du décor dans la coroplastic archaïque Proceedings of the Ist Internal. Confer. of Cypriote Studies. Lic̀ge, 241-245. 